\title{
High-Efficient Photonic Thermal Rectification with Magnetocontrollability
}

\author{
Kaiyu Wang ${ }^{1}$ and Lei Gao ${ }^{1,2^{*}}$
}

We demonstrate strong thermal rectification effect based on the thermal radiation betweentwo InSb nanospheres, with the magnetic field breaking the symmetry of system. The results reveal the thermal rectifier can achieve good efficiency in the temperature band from $330 \mathrm{~K}$ to $500 \mathrm{~K}$ and the high rectification factor is up to 100 under the temperature bias of 100 $\mathrm{K}$. Strong on- and off-resonance switching regulated by magnetic field leads to large difference of thermal flux in the forward and reverse scenarios. This study might be useful for efficient thermal management, thermal radiative insulation, and thermal information processing.

Keywords: Two-sphere; Magneto-optical; Thermal rectification

Received 29 September 2019, Accepted 24 November 2019

DOI: $10.30919 /$ esee 8 c350

\section{Introduction}

In recent years, the capabilities for controlling the heat flux by photon at the nanoscale has gained much attention due to its broad applications in heat management, photothermal conversion, ${ }^{1}$ information processing and so on. In particular, much significant progress has been made in the generation of photonic thermal devices, such as thermal rectifiers, ${ }^{2}$ thermal transistors ${ }^{3}$ and switches. ${ }^{4}$ A photonic thermal rectifier is a twobody device showing the asymmetry of radial thermal transport with respect to the temperature gradient between two contactless terminals. Compared with conventional thermal rectifiers based on nonlinear phonons or electrons transport, ${ }^{5}$ the photonic thermal rectifiers without the constraint of heat carrier speed are more advantageous to work over a wide operating bandwidth.

As one knows, photon-based thermal rectifiers were generally realized by utilizing the plane-plane geometry with dissimilar materials through the temperature-dependent optical resonance. For instance, Otey et al. proposed a photonic thermal diode consisting of two plates with $\mathrm{SiC}-3 \mathrm{C}$ and $\mathrm{SiC}$ $6 \mathrm{H}$, and significant rectification results from the interaction of temperature-dependent surface phonon polaritons. ${ }^{2}$ Later, similar geometries involving various materials are carried out to improve the efficiency of thermal rectification, such as $\mathrm{SiC}$ and $\mathrm{SiO}_{2}$ plates, ${ }^{6}$ intrinsic $\mathrm{Si}$ plate and doped $\mathrm{Si}$ grating, ${ }^{7} \mathrm{SiO}_{2}$ and $\mathrm{InSb}$ coated with graphene. ${ }^{8}$ Moreover, radiative thermal diodes were proposed thanks to the phase transitions of $\mathrm{VO}_{2}$ materials. ${ }^{9-11}$ On the other hand, much stronger rectification contrasts in thermal radiation in the two-sphere geometry were also investigated. For different sphere sizes, ultrahigh-contrast and large-bandwidth thermal rectification were shown due to the large difference in the coupling constants between dipole and quadrupole modes in the forward and reverse scenarios. ${ }^{12}$ Also, ultrahigh photonic thermal rectification was designed and the underlying mechanism lies in the prominent increase of imaginary part of the permittivity of silicon induced by thermally excited electrons at high temperature. ${ }^{13}$

Herein, we propose photonic thermal rectifier based on two nanospheres made of magneto-optical materials. The magneto-optical (MO) nanospheres supporting localized surface plasmon (LSP) resonance may excite optical bistability/tristability, ${ }^{14}$ ultrafast Fano switching,${ }^{15}$ and even provide novel mechanisms in thermal radiation, including circular heat, ${ }^{16}$ giant thermal magnetoresistance, ${ }^{17}$ photon thermal Hall effect, ${ }^{18}$ and the nonreciprocity in the threebody thermal transfer. ${ }^{19,20}$ Since the resonant mode coupling

${ }^{1}$ College of Physical Science and Technology, Soochow University, Collaborative Innovation Center of Suzhou Nano Science and Technology, Soochow University, Suzhou 215006, China

2 Jiangsu Key Laboratory of Thin Films, Soochow University, Suzhou 215006, China

*E-mail: leigao@suda.edu.cn 
between the two nanospheres can be adjusted by magnetic field, the thermal rectifier can be actively controlled without the change of structure, and it is expected to obtain high thermal rectification factor.

\section{Computational Methods}

As is shown in Fig. 1, the radiative thermal rectifier consists of two magneto-optical nanospheres ( $\mathrm{InSb}$ ) separated by a vaccum gap. In the forward temperature biased scenario, sphere I is maintained at high temperature $T_{h}$ and sphere II is at low temperature $T_{l}$. In the reverse one, the temperature of two spheres is swapped. It is evident that there is no thermal rectification between two identical spheres without the external magnetic field. In our case, we would like to apply the magnetic field sphere I only (no magnetic field is applied to the sphere II). As a result, one breaks the symmetry and may induce the thermal rectification. Actually, the thermal transfer between two magneto-optical nanospheres can be controlled and modulated in presence of the external magnetic field.

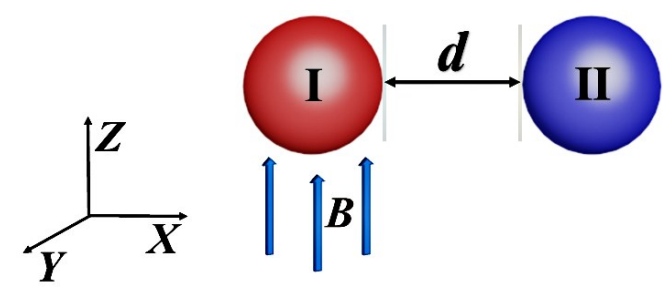

Fig. 1 The two terminals of thermal rectifier are composed of two InSb spheres kept at different temperatures. All parameters of the two spheres are the same, including radius $a=100 \mathrm{~nm}$, and they are separated by vacuum gap $d$. The magnetic field $B$ on $\mathrm{Z}$ axis is applied to sphere $\mathrm{I}$.

When sphere $\mathbf{I}$ is subjected to an external magnetic field, the permittivity tensor of the InSb adopts the form ${ }^{16}$

$$
\bar{\varepsilon}_{\mathrm{I}}=\left(\begin{array}{ccc}
\varepsilon_{1} & -i \varepsilon_{2} & 0 \\
i \varepsilon_{2} & \varepsilon_{1} & 0 \\
0 & 0 & \varepsilon_{3}
\end{array}\right),
$$

with $\quad \varepsilon_{1}=\varepsilon_{\infty}\left(1+\frac{\omega_{p}^{2}(\omega+i \gamma)}{\omega\left[\omega_{c}^{2}-(\omega+i \gamma)^{2}\right]}\right)$,

$$
\varepsilon_{2}=\frac{\varepsilon_{\infty} \omega_{p}^{2} \omega_{c}}{\omega\left[(\omega+i \gamma)^{2}-\omega_{c}^{2}\right]}, \varepsilon_{3}=\varepsilon_{\infty}\left(1-\frac{\omega_{p}^{2}}{\omega(\omega+i \gamma)}\right) .
$$

In Eq. (2), the relevant parameters are the high-frequency permittivity $\varepsilon_{\infty}=15.75$, the plasma frequency $\omega_{p}=$ $\sqrt{n(T) e^{2} /\left(m^{*} \varepsilon_{0} \varepsilon_{\infty}\right)}$, the scattering rate $\gamma(T)=e / m^{*} \mu(T)$, and the cyclotron frequency $\omega_{c}=e B / m^{*}$ with the electron's effective mass $m^{*}$ is $0.015 m_{e}$. The temperature-dependent carrier mobility $\mu(T)$ and free carries of density $n(T)$ are obtained from. ${ }^{8,21}$ As we shall show, the introduction of the temperature-dependent material parameters in magnetooptical permittivity tensor provides us the necessary conditions for thermal rectification.

Since the size is small enough compared with their thermal wavelength, two nanospheres with different temperatures can be regarded as electric dipole. ${ }^{22}$ The quasistatic polarizability for the magneto-optical sphere is described by the form $\overline{\bar{\alpha}}_{\mathrm{I}}=4 \pi a^{3}\left(\overline{\bar{\varepsilon}}_{\mathrm{I}}-\overline{\overline{1}}\right)\left(\overline{\bar{\varepsilon}}_{\mathrm{I}}+2 \overline{\overline{1}}\right)^{-1}$, where $\overline{\overline{1}}$ stands for the unit dyadic tensor. The quasistatic polarizability of isotropic sphere II can be obtained without the magnetic field, i.e., $B=0$. Here, we mention that we do not taken into account the radiative correction of the polarizability due to its minor effect for subwavelength particles.

Based on the Landauer-like formalism for two-body system, the thermal flux between particle $i$ and particle $j$ is given by ${ }^{18,19}$

$$
Q=3 \int_{0}^{\infty} \frac{d \omega}{2 \pi}\left[\Theta\left(\omega, T_{i}\right)-\Theta\left(\omega, T_{j}\right)\right] \Gamma_{i j}(\omega),
$$

where $\Theta(\omega, T)=\hbar \omega /\left(e^{\hbar \omega / h_{b} T}-1\right)$ is the average energy of a harmonic oscillator at temperature $T . \Gamma_{i j}(\omega)$ is the frequency-dependent transmission coefficient defined as

$\Gamma_{i j}(\omega)=\frac{4}{3} \operatorname{Im}\left\{\operatorname{Tr}\left[\overline{\bar{C}}_{i j}^{-1} \frac{\left(\overline{\bar{\alpha}}_{j}-\overline{\bar{\alpha}}_{j}^{\dagger}\right)}{2 i}\left(\overline{\bar{C}}_{i j}^{-1}\right)^{\dagger}\left(\overline{\bar{\alpha}}_{i}^{-1}\right)^{\dagger}\right]\right\}$.

This transmission coefficient $\Gamma_{i j}(\omega)$ resembles the transmission probability for the electrons in the Landauer formula. In Eq. (4), the $\overline{\bar{C}}$ matrix is related to electric Green's functions $\quad \overline{\bar{G}}_{i j}^{0}=\frac{e^{i k r_{i j}}}{4 \pi r_{i j}}\left[\left(1+\frac{i k r_{i j}-1}{k^{2} r_{i j}^{2}}\right) \overline{\overline{1}}+\frac{3-3 i k r_{i j}-k^{2} r_{i j}^{2}}{k^{2} r_{i j}^{2}} \frac{r_{i j} \otimes r_{i j}}{r_{i j}^{2}}\right] \quad\left(r_{i j}=\right.$ $\left|r_{i j}\right|, r_{i j}$ is the vector linking the center of dipoles $i$ and $\left.j\right)^{18}$ with

$$
\overline{\bar{C}}_{i j}=\delta_{i j} \overline{\overline{1}}-\left(1-\delta_{i j}\right) k_{0}^{2} \overline{\bar{\alpha}}_{i} \overline{\bar{G}}_{i j}^{0} .
$$

From the analysis of the quasistatic polarizability $\overline{\bar{\alpha}}_{\mathrm{I}}$, one can show that three resonant modes can be obtained from the following solutions ${ }^{16,23}$

$$
\operatorname{det}\left(\overline{\bar{\varepsilon}}_{\mathrm{I}}+2 \overline{\overline{1}}\right)=\left(\varepsilon_{3}+2\right)\left[\left(\varepsilon_{1}+2\right)^{2}-\varepsilon_{2}^{2}\right]=0 .
$$

Without the external magnetic field, the dipole resonant modes with magnetic quantum numbers $m=$ $-1,0,+1$ are degenerate in zero-field mode, whose resonant frequency $\omega_{r}(B=0, T, m=0)$ is the solution of the usual equation $\varepsilon_{3}=-2 .{ }^{24}$ However, with the existence of the magnetic field, the degeneracy between the two modes for 
$m=-1$ at the resonant frequency $\omega_{r}(B, T, m=-1)$ and $m=$ +1 at the resonant frequency $\omega_{r}(B, T, m=+1)$ is lifted. Two separated modes for $m=+1$ and $m=-1$ depend on the intensity of the magnetic field, while the $m=0$ mode does not change with the magnetic field, i.e., $\omega_{r}(B, T, m=0)=$ $\omega_{r}(0, T, m=0)$. Note that all those resonance frequencies are strongly dependent on the temperature.

We are now in a position to design the thermal rectifier with mode coupling method. ${ }^{2}$ The generation of thermal rectification effect is relevant to the magnetic field in our research system, and the magnetic field can be regarded as the switch of the thermal rectifier. The existence of separated modes caused by magnetic field makes it possible for us to flexibly couple and separate modes, which is the key factor for the efficient heat rectification.

The net heat flux between two spheres is denoted as $Q_{F}$ or $Q_{R}$ in the forward or reverse temperature biased scenario. In order to highlight the efficiency of thermal rectification, we define rectification factor $R$ as the relative variation of the heat flux in the two situations,

$$
R=\left(Q_{F}-Q_{R}\right) / \min \left(Q_{F}, Q_{R}\right) .
$$

Based on the above definition, the positive rectification $R$ means the forward heat flux is larger than the backward flux. On the contrary, the backward flux is larger than the forward one, leading to the negative rectification factor.

\section{Results and discussion}

The effect of magnetic field on thermal rectification is investigated firstly. In all calculations, the low temperature $T_{l}$ is kept at $300 \mathrm{~K}$. We plot the net heat flux in both forward and reverse biased scenarios, and rectification factor $R$ as a function of the magnetic field $B$ in Fig. 2(a). We observe that the maximal forward thermal flux appears at magnetic field of $5.6 \mathrm{~T}$ and the maximal reverse thermal flux appears at $B=$ 2.4 T. Consequently, near these two magnetic fields, the system reaches maximal positive (or negative) rectification factor of 196 (or -12.8). The rectification factor predicted here is much larger than the theoretical values of 5.14 reported in ${ }^{12}$ and 16.75 in. ${ }^{7}$ There are two reasons for our good thermal rectification, one is that the semiconductor material we chose supports narrow-banded near-field heat transfer, ${ }^{2}$ the other is we can adjust the coupling and separation of modes with the magnetic field easily.

In addition, the rectification factor undergoes a change from negative to zero to positive with increasing magnetic field intensity and the crossover point for the reversal of the direction in our rectifier is $B=3.1 \mathrm{~T}$. Such reversal was found in quantum one-dimensional systems too. ${ }^{25}$

In order to understand the physical mechanisms behind it, we investigate the spectral transmission in Fig. 2(b-f). The spectral transmission is the same in the forward and reverse biased scenarios for zero magnetic field, as shown in Fig. 2(b). As the temperature increases, the concentration
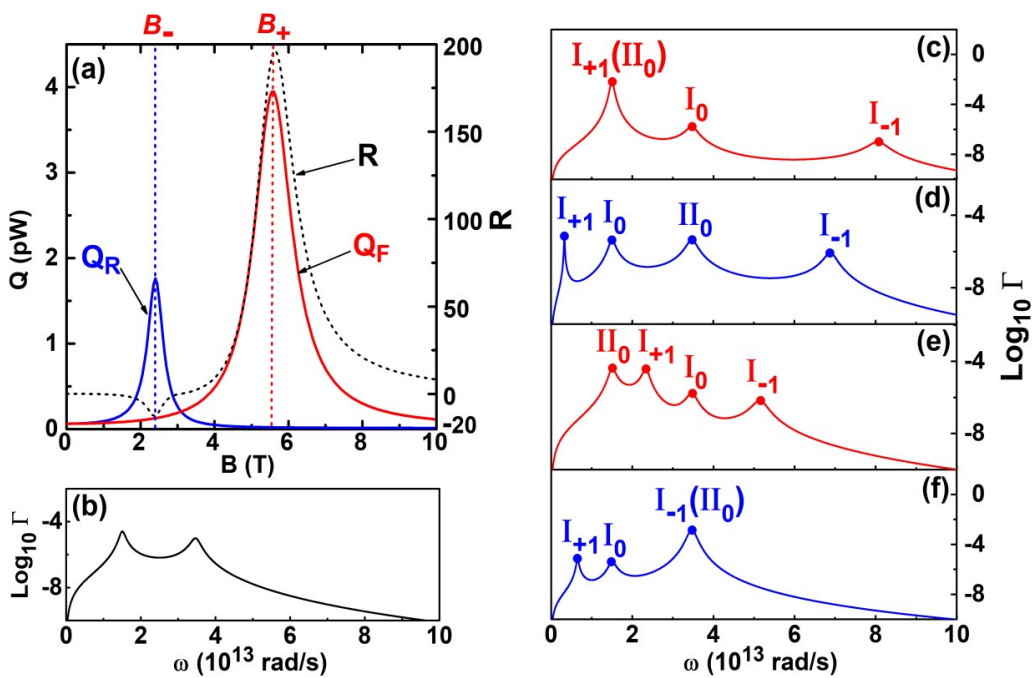

Fig. 2 (a) Net heat flux $Q$ and rectification factor $R$ as a function of the magnetic field $B$ for $T_{h}=400 \mathrm{~K}$ and $\mathrm{d}=2 \mathrm{a}=200 \mathrm{~nm} . B_{+}\left(B_{-}\right)$ denotes the magnetic field corresponding to maximal forward (reverse) thermal flux. Spectral transmission ae shown in (b) for zero magnetic field. (c) and (d) Spectral transmission for magnetic field $B_{+}=5.6 \mathrm{~T}$ in the forward and reverse biased scenarios, respectively. (d) and (f) Spectral transmission for $B_{-}=2.4 \mathrm{~T}$ in the forward and reverse biased scenarios. Each peak is labeled with the corresponding mode above them. 
of free electrons in the nanosphere rises, resulting in the blue-shift of the dipole resonant frequency of zero field mode. In the forward biased scenarios, the dipole resonant frequencies of the sphere $\mathbf{I}$ at high temperature $T_{h}$ and $\mathbf{I I}$ at low temperature $T_{l}$ are, respectively, located at $3.5 \times$ $10^{13} \mathrm{rad} / \mathrm{s}$ and $1.5 \times 10^{13} \mathrm{rad} / \mathrm{s}$. In the reverse biased case, the resonant frequencies of two nanospheres are exchanged.

For the magnetic field applied to sphere $\mathbf{I}$ at temperature $T$, two magnetic-field-dependent modes separate from the degenerate state, and hence there are three dipole resonant frequencies $\omega_{r}(\mathrm{~B}, \mathrm{~T}, \mathrm{~m}=-1), \omega_{r}(B, T, m=$ $+1), \omega_{r}(B, T, m=0)$. On the other hand, the sphere II at temperature $T$ with zero magnetic field only shows one resonant frequency $\omega_{r}(B=0, T, m=0)$. Since those four resonant frequencies are nonoverlapping, one observes four resonant peaks generally in the spectral transmission in the reverse biased scenario, which is called in the off-resonance state. ${ }^{26}$ However, for the applied magnetic field of $B_{+}=5.6 \mathrm{~T}$, one just achieves the on-resonance state at which the overlapping resonant frequencies take place, i.e., $\omega_{r}\left(\mathrm{~B}_{+}, \mathrm{T}_{h}, \mathrm{~m}=+1\right)$ equals $\omega_{r}\left(B=0, T_{l}, m=0\right)$ in the forward biased scenario, resulting in three resonant peaks with one strong transmission (see Fig. 2(c)). The strong transmission peak implies the strong forward heat flux, resulting from the on-resonance state. Compared with the reverse heat flux in the off-resonance state, the forward heat flux will be many times larger, resulting in the high positive rectification factor.

Similarly, when the magnetic field $B_{-}=2.4 \mathrm{~T}$ is applied to sphere $\mathbf{I}$, in the reverse biased scenario, the resonant high-frequency $\omega_{r}\left(B_{-}, T_{l}, m=-1\right)$ coincides with the resonant frequency $\omega_{r}\left(\mathrm{~B}=0, T_{h}, m=0\right.$ ) of sphere II (see Fig. 2(f)). As a consequence, the system is in on-resonance state in the reverse biased scenario, leading to much larger $Q_{R}$. On the other hand, two nanospheres are in off resonance state in the forward biased scenario, resulting in small $Q_{F}$. Such on- and off-resonance transformations make the forward heat flux far smaller than the reverse one. Therefore, we can obtain the negative thermal rectification factor. Notably, due to the contribution from the low frequency $m=+1$ mode to the thermal transfer is much greater than that of high-frequency $m=-1$ mode in the near field region, ${ }^{16}$ the maximal positive rectification factor tends to be larger than maximal negative one. The mechanism for our photonic thermal rectification lies in when the magnetooptical materials with temperature-dependent parameters are taken into account, it is possible to realize the onresonance state in the forward/reverse biased scenario and the off-resonance state in the reverse/forward one, and yield high thermal rectification. This should be in contrast with the work in Raf. [27], in which the radiative thermal diode is composed of two magneto-optical spheres, located above the planar magnetic-optical substrate. The mechanism is based on the asymmetry with respect to the propagation direction of evanescent waves confined at the surface of the magneto-optical media.

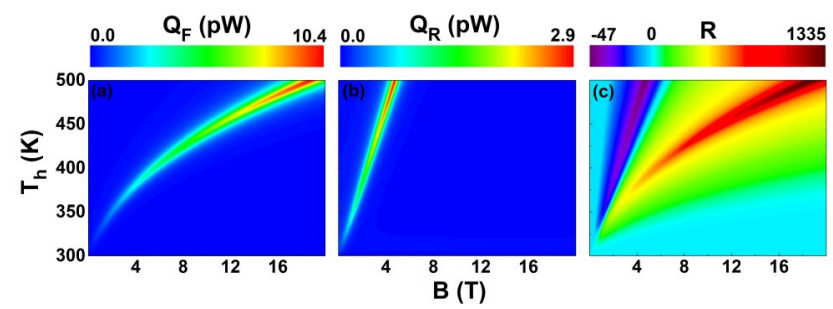

Fig. 3 (a) Forward Heat flux $Q_{F}$, (b) Reverse heat flux $Q_{R}$, and (c) Corresponding rectification factor $R$ as a function of temperature $T_{h}$ and magnetic field $B$ for $d=200 \mathrm{~nm}$.

Next, we consider the influence of different temperature bias on rectification. In Fig. 3(a), strong forward heat flux is observed on the on-resonance condition, at which the resonance frequency of $\mathrm{m}=+1$ mode $\omega_{r}\left(B_{+}, T_{h}, m=+1\right)$ coincides with the zero-field mode $\omega_{r}\left(B=0, T_{l}, m=0\right)$. In other words, for each $T_{h}$, there is an optimal magnetic field $B_{+}$, at which the forward heat flux takes an maximum. Therefore, compared with other thermal rectifiers based on mode-coupling, we only need to change the magnetic field with the temperature difference to make the system on resonance at different temperature bias. Since the zero field mode is shifted toward the higher frequency as $T_{h}$ increases, stronger magnetic field $B_{+}$is needed to satisfy the on-resonance condition. Actually, because of this correspondence between $T_{h}$ and $B_{+}$, our device exhibits negative differential thermal conductance, ${ }^{12}$ too. For example, for $B=5.6 \mathrm{~T}$, the forward net heat flux takes the maximal value at $T_{h}=400 \mathrm{~K}$. As $T_{h}$ increases further, the net thermal flux is reduced, due to the weak coupling between the two spheres. As a consequence, one yields the negative differential thermal conductance. In Fig. 3(b), similar behavior is also reflected by the strong backward flux due to the onresonance state at which the resonant frequency $\omega_{r}\left(B_{-}, T_{l}, m=-1\right)$ coincides with $\omega_{r}\left(B=0, T_{h}, m=0\right)$. The great contrast between these two pictures indicates strong rectification.

Thus, the positive and negative branches representing the strong rectification are presented in Fig. 3(c). From $330 \mathrm{~K}$ to $500 \mathrm{~K}$, the obvious phenomenon of thermal rectification can be observed. For higher $T_{h}$, the thermal rectification effect is better, and the two branches are further separated. Our numerical calculation shows in the case of $T_{h}=500 \mathrm{~K}$, the rectification factors corresponding to the magnetic field $B_{+}=18.80 \mathrm{~T}$ and $B_{-}=4.75 \mathrm{~T}$ are 1333.68 and -46.78 , 
respectively. For the low emitter temperature of $330 \mathrm{~K}$, the rectification factor for $B_{+}=1.05 \mathrm{~T}$ and $\mathrm{B}_{-}=0.75 \mathrm{~T}$ is only 1.90 and -0.32 , respectively.

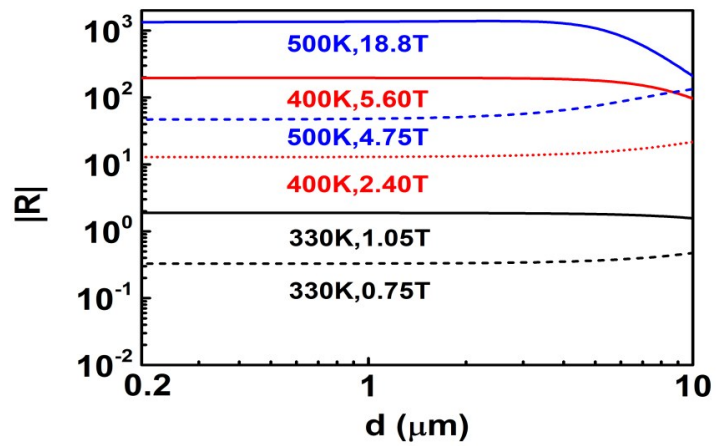

Fig. 4 Rectification factor $|R|$ vs the vacuum gap $d$ for magnetic field $B_{+}$(solid line) or $B_{-}$(dashed line) and different $T_{h}$.

In the end, we would like to study the evolution of $R$ with the separation $d$, as shown in Fig. 4. It is evident that the rectification factor are less sensitive to the gap variation within $1 \mu \mathrm{m}$. The two-sphere system shows a stark contrast to the planar structure whose rectification factor severely decreases with the increasing of gap distance. ${ }^{2,8}$ When $d$ is larger than $5 \mu \mathrm{m}$, the contribution of $\mathrm{m}=+1$ mode to transmission decreases and that of $m=-1$ mode increases, leading to the obvious change in the rectification curves. This robustness with the distance is quite helpful for us to design the efficient photonic thermal diodes.

Here, we mention that although our device can also achieve a high rectification factor over long distance, the weak heat flux limits the practical use of the thermal rectifier. The thermal radiation of the two spheres attenuates by several orders of magnitude at a long distance. ${ }^{16}$ In most cases, the heat flux usually only reach below $10^{-15} \mathrm{~W}$ near a $1.5 \mu \mathrm{m}$ gap. For a longer distance, the small amount of heat makes against rapid thermal exchange in a wider gap.

\section{Conclusions}

In summary, a photonic thermal rectifier with magneto-optical $\mathrm{InSb}$ is proposed. Due to the mode coupling can be actively tuned by changing the magnitude of the external magnetic field, the thermal rectifier shows strong rectification effect and the rectification factor has a large adjustable range. To one's interest, thermal rectifier proposed here can achieve high efficiency even under small temperature bias, and the rectification does not decrease significantly with gap distance. Results obtained from this study may have wide applications in electronics cooling, energy conversion, thermal logical circuits, and so on.

\section{Conflict of interest}

There are no conflicts to declare.

\section{Acknowledgements}

This work was financially supported by PAPD of Jiangsu Higher Education Institutions; National Natural Science Foundation of China (11774252); National Science of Jiangsu Province (BK20161210); the Qing Lan project; "333" project (BRA2015353).

\section{References}

1. Y. Ren, Q. Chen, H. Qi and L. Ruan, ES Energy Environ., 2019, 3, 74 79.

2. C. R. Otey, W. T. Lau and S. Fan, Phys. Rev. Lett., 2010, 104, 154301.

3. P. Ben-Abdallah and S. A. Biehs, Phys. Rev. Lett., 2014, 112, 044301.

4. O. Ilic, N. H. Thomas, T. Christensen, M. C. Sherrott, M. Soljačić, A. J. Minnich, O. D. Miller and H. A. Atwater, ACS Nano, 2018, 12, 24742481.

5. B. Zhang, P. Mao, Y. Liang, Y. He, W. Liu and Z. Liu, ES Energy Environ., 2019, 5, 37-55.

6. K. Joulain, Y. Ezzahri, J. Drevillon, B. Rousseau and D. D. S. Meneses, Opt. Express, 2015, 23(24), A1388-A1397.

7. J. Shen, X. Liu, H. He, W. Wu and B. Liu, J. Quant. Spectrosc. Radiat. Transfer, 2018, 211, 1-8.

8. G. Xu, J. Sun, H. Mao and T. Pan, J. Quant. Spectrosc. Radiat. Transfer, 2018, 220, 140-147.

9. P. Ben-Abdallah and S. A. Biehs, Appl. Phys. Lett., 2013, 103, 191907.

10. A. Ghanekar, J. Ji and Y. Zheng, Appl. Phys. Lett., 2016, 109, 123106.

11. S. Jia, Y. Fu, Y. Su and Y. Ma, Opt. Lett., 2018, 43(22), 5619-5622.

12. L. Zhu, C. R. Otey and S. Fan, Phys. Rev. B, 2013, 88, 184301.

13. S. Wen, X. Liu, S. Cheng, Z. Wang, S. Zhang and C. Dang, J. Quant. Spectrosc. Radiat. Transfer, 2019, 234, 1-9.

14. W. J. Yu, H. Sun and L. Gao, Opt. Express, 2016, 24, 22272-22281.

15. W. J. Yu, P. J. Ma, H. Sun, L. Gao and R. E. Noskov, phys. Rev. B, 2018, 97, 075436 .

16. A. Ott, P. Ben-Abdallah and S. A. Biehs, Phys. Rev. B, 2018, 97, 205414.

17. I. Latella and P. Ben-Abdallah, Phys. Rev. Lett., 2017, 118, 173902.

18. P. Ben-Abdallah, Phys. Rev. Lett., 2016, 116, 084301.

19. A. Ott, R. Messina, P. Ben-Abdallah and S. A. Biehs, J. Photonics Energy, 2019, 9, 032711.

20. L. Zhu and S. Fan, Phys. Rev. Lett., 2016, 117, 134303.

21. F. Fan, C. Z. Xiong, J.R. Chen and S. J. Chang, Opt. Lett., 2018, 43, 687690.

22. R. M. Abraham Ekeroth, A. García-Martín and J. C. Cuevas, Phys. Rev. $B, 2017,95,235428$.

23. R. M. Abraham Ekeroth, P. Ben-Abdallah, J. C. Cuevas and A. GarcíaMartín, ACS Photonics, 2018, 5, 705-710.

24. R. P. Devaty, Phys. Rev. B, 1988, 38, 7972-7979.

25. L. Zhang, Y. H. Yan, C. Q. Wu, J. S. Wang and B. Li, Phys. Rev. B, 2009, 80, 172301

26. L. Tang and M. Francoeur, Opt. Express, 2017, 25, A1043-A1052.

27. A. Ott, R. Messina, P. Ben-Abdallah and S.A. Biehs, Appl. Phys. Lett., 2019, 114, 163105 .

Publisher's Note Engineered Science Publisher remains neutral with regard to jurisdictional claims in published maps and institutional affiliations. 